\title{
Performance Comparison of Low-Latency Anonymisation Services from a User Perspective
}

\author{
Rolf Wendolsky, Dominik Herrmann, and Hannes Federrath \\ University of Regensburg, 93040 Regensburg, Germany
}

\begin{abstract}
Neither of the two anonymisation services Tor and AN.ON clearly outperforms the other one. AN.ON's user-perceived QoS is generally more consistent over time than Tor's. While AN.ON's network latencies are low compared to Tor, it suffers from limitations in bandwidth. Interestingly, Tor's performance seems to depend on the time of day: it increases in the European morning hours. Utilising AN.ON's reporting of concurrently logged-in users, we show a correlation between load and performance. The reported number of users should be adjusted, though, so that it serves as a better indicator for security and performance. Finally, the results indicate the existence of an overall tolerance level for acceptable latencies of approximately 4 seconds, which should be kept in mind when designing low-latency anonymisation services.
\end{abstract}

\section{Introduction and Motivation}

Several anonymisation services for low-latency communication have grown up from research projects recently: among them are the well-known systems AN.ON [3] and Tor [17. This paper focuses on the performance of the services for web surfing from a user perspective.

Although AN.ON and Tor are based on common building blocks (e. g. so called mixes [6], which relay multiply encrypted traffic from a client to a server), they differ in various technical attributes such as structure, threat model and application range. AN.ON uses a limited set of cascades, each consisting of predefined mixing nodes. In contrast, Tor relies on a large amount of nodes from which random circuits are constructed in real-time. As the user base is usually hundreds or thousands of times bigger than the amount of nodes used for relaying traffic, performance issues may arise.

It has been shown that performance, especially latency, is an anonymityrelevant parameter [1]. We can assume that many users are not able to evaluate the real security of an anonymisation service [5]. Therefore, their decision to use a specific service may highly depend on its overall performance: Only few people are willing to use a slow service, and, regardless of any sophisticated cryptographical techniques, such a service might not provide any anonymity at all. Consequently, the performance from a user perspective might serve as an important indicator for the overall quality. Moreover, performance evaluations can be used to identify characteristics of the different approaches, and - obviously - they allow the evaluation of tuning measures.

N. Borisov and P. Golle (Eds.): PET 2007, LNCS 4776, pp. 233253, 2007. 
In this paper, we will provide an empirical study regarding the relation between performance and the number of concurrent users. Based on that we will present the results of a comparison of AN.ON and Tor from a user perspective and try to explain the source of any differences found. We will show that a naïve comparison of average throughputs and delays is hardly sufficient, but conclusions can be drawn with the help of inferential statistics nevertheless. Our results indicate the existence of an overall performance threshold. This means that users are not willing to use a service which fails to meet this threshold.

We will introduce the evaluation scenarios for our performance tests in section 2 and present our methodology for data collection in section 3 . Section 4 contains a short description of the statistical methods used during analysis. The results of our evaluation of AN.ON and Tor are presented in section 5 . We suggest areas for future research in section [6, while section 7 summarizes our findings.

\section{Performance Indicators and Evaluation Scenarios}

In this section we will present the relevant performance indicators and our evaluation scenarios. For the performance evaluation of the anonymisation services, we simulate the behaviour of a typical WWW-user who (1) requests web sites and (2) downloads files. We identified two performance indicators, namely latency and bandwidth.

The bandwidth (KBytes/s) indicates how fast data packets may be transmitted on a communication channel. The latency (milliseconds) corresponds to the roundtrip time of a network packet. Ideally, the latency is independent from the bandwidth. For large files it is almost irrelevant, whereas retrieving a web site (with many small objects) can be slowed down by high latencies substantially.

In order to profile the aforementioned indicators, we set up different scenarios. A scenario is characterised by two parameters: type of simulation and URL language. The type of simulation is either (1) a test with different web sites containing a (large) number of small objects $\{\mathrm{WEB}\}$, or $(2)$ a test with fixed-size downloads $\{\mathrm{DL}\}$. The separation into different $U R L$ languages is a heuristic method to measure system performance in a local area, e. g. Germany, or world-wide. For our research, we split the tests into German $\{\mathrm{DE}\}$ and English $\{\mathrm{EN}\}$ content language. While the English pages can be used for a fair comparison of different anonymisers, the German sites allow profiling the AN.ON service from a local perspective 1 The URLs were chosen from the most popular web sites according to Alexa [2] and the downloads according to downloads.de/downloads.com respectively (cf. table 7 ). Table 1 lists the basic scenarios.

\section{Data Collection Methodology}

In this section we will describe our methodology for collecting performance data from anonymisation services based on an example of the Tor network and

\footnotetext{
${ }^{1}$ All current AN.ON servers reside in Germany, whereas Tor is distributed throughout the world.
} 
Table 1. General attributes of the basic scenarios

\begin{tabular}{|c|c|c|c|c|}
\hline \multirow{2}{*}{$\begin{array}{r}\text { Simulation } \\
\text { Language }\end{array}$} & \multicolumn{2}{|c|}{ WEB } & \multicolumn{2}{|c|}{$\overline{\mathrm{DL}}$} \\
\hline & $\mathrm{DE}$ & $\mathrm{EN}$ & $\mathrm{DE}$ & EN \\
\hline Total URLs / scenario & 11 & 14 & 3 & 3 \\
\hline Average requests / scenario & 398 & 309 & 3 & 3 \\
\hline Average requests / URL & 33.17 & 20.6 & 1 & 1 \\
\hline Average KBytes / scenario & 1267 & 987 & 1520 & 1702 \\
\hline Average KBytes / URL & 105.58 & 65.8 & 506.67 & 567.33 \\
\hline
\end{tabular}

AN.ON. We will start off with an overview of our evaluation setup and the evaluated services. The major part of this section will present our data quality measures.

\subsection{Test Suite Overview}

There are some free tools available to measure proxy or server performance 10 15. Unfortunately, they proved not suitable for the evaluation of anonymisation services. They focus on other applications and consequently lack important features such as failure tolerance. In the end, we decided to write a test suite specifically designed to meet our needs.

As we evaluate the services from a user perspective, the two performance parameters mentioned, bandwidth and latency, cannot be determined exactly: There are too many influences not under our control. Therefore, we approximate the performance of the services with the help of the two observable parameters throughput and initial delay. The throughput is calculated by dividing the amount of received bytes by the time needed for the data transmission. The initial delay is the time difference between sending the HTTP request and receiving the first chunk of the response.

Our test suite perfeval 2 is written in Perl (about 2.500 lines of code) 3 . The scripts retrieve a set of URLs via HTTP (non-recursively) and calculate throughput and initial delay for each HTTP request. All recorded data of a session is aggregated into a test case.

We utilise the Perl library LWP::ParallelUA [12] which can handle simultaneous connections. Thus, we are able to simulate the behaviour of a web browser: First, perfeval downloads the HTML page, and then it fetches all the embedded objects in parallel. In order to prevent proxies or web caches from influencing the results we send a Cache-Control:no-cache HTTP header 14 along with the request.

\footnotetext{
${ }^{2}$ We were running the test suite on two WindowsXP workstations with ActivePerl v5.8.7.815 1. The workstations were connected to the Internet directly and had public IP addresses.

3 http://www.jondos.de/downloads/perfeval.zip
} 


\subsection{Scope of the Evaluation}

Table 2 lists the three services we evaluated with perfeval. In the rest of this paper we will refer to them with the presented acronyms. We also use a control connection (DIRECT) for assessing the performance of the Internet connection used during testing.

Table 2. Evaluated systems

\begin{tabular}{ll}
\hline DIRECT & Direct web access without any proxy \\
TOR & Tor client v0.1.0.16, Privoxy v3.0.3 \\
DD & AN.ON cascade Dresden-Dresden (JAP v00.05.078) \\
CCC & AN.ON cascade Regensburg-CCC (JAP v00.05.078) \\
\hline
\end{tabular}

Privoxy was configured with the option toggle 0 in order to disable all of its filtering rules. The two mentioned AN.ON cascades were chosen because of high stability and high number of users at the time when we started the test 4 The test run started on February 15 2006, 6:00 p. m., and ended on February 26 2006, 11:59 a.m. (both Berlin local time 5) by manual interruption. Thus, we got test data for 10 complete days and 18 hours, that corresponds to 258 hour-based test cases for each combination of scenario parameters and tested systems. We therefore have 4128 test cases altogether.

For the scope of this article an individual web site or a file download is represented by its URL. Each URL may lead to a number of HTTP requests: Typically, a web sit causes additional requests (for the HTML page and all its embedded objects), whose number typically differs over time, whereas a download causes exactly one HTTP request.

\subsection{Data Quality Measures}

In order to get statistically utilisable results for measuring the tested services, the collected data should not be considerably influenced by

(a) external factors jeopardizing the validity of the test cases like downtimes of the network, downtimes and failures of services, HTTP errors reported by web sites, and errors in the evaluation software itself,

(b) bias introduced by the observation itself like concurrent tests on the same anonymisation service, concurrent test requests of the same resource, and performance fluctuations on the computer where the test software runs,

(c) influences through fluctuations during the test like performance fluctuations of requested resources and fluctuations of the total amount of requested data,

\footnotetext{
${ }^{4}$ At that time the remaining two AN.ON cascades were used for testing purposes only, and were neither stable in structure nor in code.

${ }^{5}$ Note that Germany has one single time zone.
} 
(d) performance tampering through HTTP redirects,

(e) performance limit introduced by the Internet conection,

(f) varying performance throughout the day.

These influences have to be mitigated before and during the test. After that, the collected data must be examined for influences by the aforementioned factors. If at least one of those has a non-negligible influence, the corresponding data is probably not usable for any statistical analysis. We assume an influence as nonnegligible if the ratio of (possibly influencing) "critical" cases to "good" cases is higher than $5 \% 6$

In short, we found that our test data is of high quality regarding these measures. A more detailed description of our approach to measure data quality is presented in the following sections.

External factors. Single erroneous test cases resulting from a bad implementation of the test software may be discovered by looking for extreme values in the number of HTTP requests (which should be the same for each test case), the initial delay and the throughput.

HTTP errors, service failures, network and service downtimes may lead to missing or unintentionally influenced cases. For each unsuccessful HTTP request (i. e., the status code of the HTTP indicates a failure), we have to determine whether the source of the problem is the webserver or the network (i. e., the anonymisation service or the Internet connection). We will refer to the former as errors, to the latter as failures. This differentiation is important to measure the "quality" of an anonymisation service. Our software implements a sophisticated algorithm to differentiate errors from failures:

An unsuccessful HTTP request will be flagged as an error, if all of the following conditions apply immediately after the HTTP response has been received:

- a connection to the webserver/proxy can be established successfully

- a HTTP test request can be sent over the network

- a corresponding HTTP response is received

- the HTTP status code is not 200 OK (or something similar)

- the HTTP status code is not 502 Service temporarily overloaded, or 503 Gateway timeout

Otherwise, the unsuccessful request is probably a failure, but further examinations are necessary. This is especially true for responses with status codes 502 and 503, which can be issued by the webserver as well as by the proxy server. If the webserver is the originator, the request should be flagged as error, otherwise as failure. Timeouts, i. e., delays exceeding 60 seconds, are the most common type of failures.

Table 8 lists the number of cases missing either due to software errors or because of network or service downtimes. Compared to the total number in the

${ }^{6}$ Note that this is a heuristic approach. The quality measures are ratios and not probabilities as in statistical tests. 
sample, they are negligible. It also shows that almost all failures occur for DD, but as less than $5 \%$ of all requests are affected, we still treat external influences as negligible. This finding indicates hardware or network problems on the AN.ON DD cascade, though. Its operators have not been aware of that until now.

The number of errors is uncritical for all but one case: the error ratio on the CCC cascade for English downloads is about $9 \%$. That means that a lot of downloads were skipped, probably due to service-specific blockings by the web site operators (e.g. by blacklisting the IP of the last mix of the cascade). Nevertheless, this influence is limited to reducing the sample size for this service.

Bias introduced by the observation itself. The tests for web surfing / downloads together were composed to be completed in less than 30 minutes for each language. In order to force comparable and periodic hour-of-day-based time intervals from 0 to 23 (Berlin local time), we put a hard limit of 60 minutes on the total duration of a language test. For each test case, all URLs were processed sequentially so that no interference between them was possible7. As the DE and EN tests should not interfere with each other, we performed these test cases on two separate machines, the latter one starting with a time offset of 30 minutes. Figure 1 shows the course of events during the performance evaluation.

Table 8 shows that the hard limit of one hour was never reached in our experiment and that a 30-minute-overlapping did not occur more often than in $5 \%$ of the test cases. These influences are therefore not seen as critical.

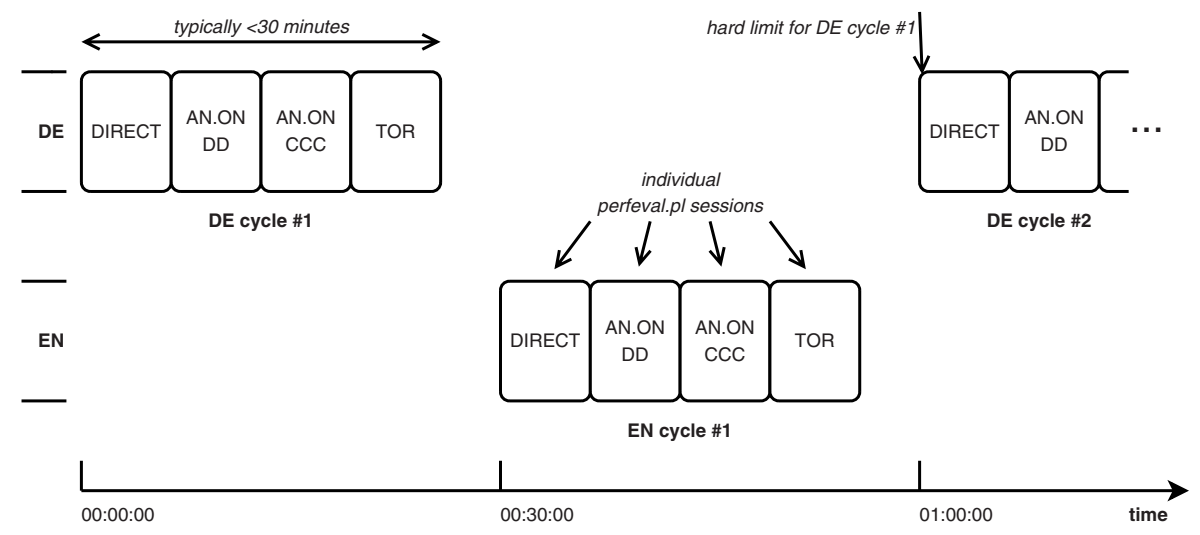

Fig. 1. Test sequence for performance evaluation

Influences through performance fluctuations. In order to avoid performance influences from slow web servers that could lead to wrong conclusions in the analysis, the measurements of the individual URLs are aggregated into one test case for each scenario. Accordingly, we do not try to evaluate the service

\footnotetext{
${ }^{7}$ Note that HTTP requests for each requested web site are done concurrently, but
} this is what a typical web browser would do as well. 
performance regarding single URLs (although this would be possible with our result files, of course).

Another possible influence is related to the amount of data received in each test case. To make the cases of one scenario comparable, they should be of equal size. We compared the median and the interquartile range 8 (IQR) of the downloaded bytes for each service with the median and IQR of all services to analyse this influence.

Table 8 shows that the ratios of all medians are negligible. Although there are some problems with English downloads (causing a huge IQR ratio for the CCC cascade), they do not affect the median. Therefore, our analysis suggests that we have indeed collected similar amounts of data for the different services.

Note that measuring performance fluctuations within the infrastructure of the anonymisation service is beyond the scope of this paper. In particular, we are not trying to measure the performance of individual nodes or one anonymity service as a whole. For Tor, we have to trust the node selection algorithm of its client software - we are looking at performance from a user perspective after all.

HTTP redirects. Our evaluation software honours HTTP 301 and 302 redirect status codes. Although this behaviour is necessary for the imitation of a web browser, it introduces a new challenge: Our test might be influenced by serverside redirects (geolocation), which would undermine the geographic separation introduced by the URL language scenario parameter.

It is rather difficult to rule out this influence completely as we cannot control the behaviour of the web servers. Of course, our software does not send any Accept-Language headers which would give away any information about its location or preferred language, nor does it interpret JavaScript code in the HTML pages which could be used to query language-specific browser attributes. But there are still more sophisticated ways for geolocation, for example by querying the WHOIS database for the IP address of the sender of the HTTP request. Obviously, it is impossible to fully prevent a webserver from delivering adapted versions of the requested content to the client. It has been observed that Tor (with its world-wide network of exit nodes) is subject to this phenomenon [18]. We screened the evaluation data to make sure that no geolocation was employed, though.

Note that language adaption is not as big a problem as it seems. HTTP requests which are automatically being redirected to a server located in close vicinity of the client are a far more intriguing threat. We have examined the URLs for the $[\mathrm{EN}]$ scenario and could not find any indication that this form of redirection was employed by any web site. Of course, some sites utilise round robin DNS entries in order to distribute the load on several webservers (e.g. google.com). But such procedures shouldn't affect the performance evaluation because their influence is averaged by the large amount of test cases.

Performance limit introduced by the Internet connection. If the local area network suffers from performance fluctuations, it may influence the observed

${ }^{8}$ The interquartile range is the difference of the upper $75 \%$ and the lower $25 \%$ quartile. It is a robust measure for the standard deviation of frequencies. 


$$
\begin{aligned}
I\left(S_{t}\right) & = \begin{cases}0 & \text { if } T h_{\text {crit }}\left(S_{t}\right) \geq 0, \text { small or no influence } \\
1 & \text { if } T h_{\text {crit }}\left(S_{t}\right)<0, \text { possible high influence }\end{cases} \\
\text { w.r.t } & \\
T h_{\text {crit }}\left(S_{t}\right) & =T h\left(\operatorname{DIRECT}_{t}\right)-T h\left(S_{t}\right)-\frac{I Q R_{B}(\mathrm{DIRECT})}{2} \\
\text { where } & \\
S & \in\{\text { DIRECT, TOR, DD, CCC }\} \\
t & :=\text { time (day and hour }) \\
S_{t} & :=\text { test case of } S \text { at the time } t \\
I Q R_{B}(\operatorname{DIRECT}) & :=\text { Interquartile range of throughput of DIRECT } \\
T h\left(S_{t}\right): & =\text { measured throughput of } S_{t} \\
T h_{\operatorname{crit}}\left(S_{t}\right) & :=\text { critical throughput of } S_{t} \\
I\left(S_{t}\right) & :=\text { possible influence of DIRECT on } S_{t}
\end{aligned}
$$

Fig. 2. Evaluating performance influences of the network connection

data as well. Network-caused performance breaks in all systems could be misinterpreted as a common attribute. For example, if the network is not faster than the slowest anonymisation service, all systems would look the same. There is no influence if the local area netwok offers better performance than the fastest system at all times.

The basic idea to estimate the possible influence of the network (DIRECT) is to analyse all single test cases of all tested systems for this possible influence. We call the ratio of the number of all cases with a non-negligible influence to the total number of cases critical influence ratio. If this ratio is, for a scenario, higher than $5 \%$, we call the influence of the network on the scenario non-negligible. Otherwise, we assume that there is no influence of the network on this scenario.

To calculate a level of non-negligibility, we suggest to evaluate all test cases by their throughput, separately for each scenario, by the formula presented in figure 2. This approach basically calculates the difference between the throughput measured for the network at a given hour and the throughput of a given test case in this hour. As a measure for the standard deviation of the network's bandwidth, we also provide the interquartile range for its througput. We subtract half of its value, as only the diminishment of the network's bandwidth is critical, and call the resulting value critical throughput for this test case. If the critical throughput is greater than zero, we assume a low possibility for network interference. Otherwise, the network influence is assumed to be non-negligible for this test case. As shown in table 8 (critical throughput influence ratio), we found a non-negligible network influence for 5 out of 12 scenarios. This means that care must be taken when these scenarios are analysed, as at least some clipping phenomend 9 are expected.

${ }^{9}$ Clipping means that some performance curves will have a hard break in the peaks. 
Varying performance throughout the day. An anonymity service saturated with a big and distributed user group is expected to show a normal distribution in user numbers, bandwidth and latency for each hour and day. In reality, though, the user groups may be heterogenous and therefore have a strong influence on performance over time. Before statistically analysing and comparing services, it is therefore useful to exploratively identify time-dependend trends in the user behaviour.

During the performance evaluation we retrieved the real-time number of concurrent users provided by the AN.ON services for further analysis. We identified two major trends:

1. The user numbers seem to follow a sinusoidal curve with vertex at 11 a. $\mathrm{m}$. (cf. figure 5). Given that most users of AN.ON are located in Europe 8, this means that the majority of them is using the service during the day and not during the night.

2. The variables throughput and delay seem to be normally distributed between 1 p. m. and 9 p.m. Therefore, the influence of varying loads on the AN.ON services is expected to be minimal in that time period.

Therefore, we decided to introduce a new scenario parameter daytime to simplify the comparison of AN.ON with Tor, which is more equally distributed over the whole day. daytime has the values morning (M) and afternoon (A), defined as the hour-of-day intervals 1-9 a.m. and 1-9 p.m. (Berlin local time). All test data from the remaining time periods was discarded.

\section{Statistical Methodology for Analysis and Comparison}

For distinguishing differences in our sample from "random noise", we performed thorough statistical analyses. This section provides a short explanation of the statistical background needed to understand the results presented in section 5.

\section{$4.1 \quad$ t-tests}

In order to compare two samples, we use Student's t-test, which is very robust against violations of the normality assumption. In this paper we will use t-tests to compare the mean value of a given parameter (i. e., throughput or delay) of two samples (i.e., two anonymisation services). The t-test checks whether the means of the tested parameters differ significantly (hypothesis $H_{1}$ ).

t-tests can only be applied under the following assumptions [16]:

1. normal distribution of data

2. homogeneity of variances

3. independent, randomly selected samples

The last assumption is already addressed by the data quality measures mentioned in section 3.3 . As we cannot expect the data in our samples to be normally 
distributed, we employ the Kolmogorov-Smirnov test. If the result of this test is significant, the data of the sample is not normally distributed and the t-test may draw incorrect conclusions. Similarly, the equality of the variances is proven with the Levene test. Even if the Levene test shows significantly differing standard deviations, the t-test can still be applied. In this case a modified version of the t-test has to be applied, though.

In the following sections the results of the the t-tests are shown in the column labelled "Sides". The higher the number of asterisks $(*, * *, * * *)$, the more significant is the evaluated difference of mean values. A dash (-) indicates that the test found no significant difference (e.g. table 3 ).

\subsection{Regression Analysis}

We analyse possible correlations of two or more metric parameters by a Linear Regression Analysis. It tests the assumption of a linear correlation between the dependend parameter $y_{i}$ and the independent parameters $x_{i}$ of the form

$$
\hat{y}_{i}=b_{0}+\sum_{j=1}^{m} b_{j} x_{i j}
$$

for all test cases $i=1,2, \ldots, n$ and the independent parameters $j=1,2, \ldots, m$. In the following sections the confidence in the regression analysis is shown in the row labelled "Terms". The higher the number of asterisks $(*, * *, * * *)$, the more significant is the estimated influence of the parameter (cf. table 6).

In order to be able to perform a regression analysis, the basic assumptions of linearity, independence, homoscedasticity and normality must be fulfilled for the data [7].

\section{Evaluation}

As mentioned earlier we decided to split the gathered data points into two data sets according to the time of day. The graphs in figures 5 and 6 show that user numbers, delay, and throughput follow a typical course for the two AN.ON services: between 1 p. m. and 9 p. m. the curves are approximately at the same level, whereas they resemble a quadratic function with a minimum at about 5 a. m. between 1 a. m. and 9 a. m. For the comparison of the services, we focus on the first of these periods which we call 'afternoon', as the AN.ON cascades are obviously not under full load during the latter one - most users are asleep during the 'morning' hours (cf. figure 6). Combining both the morning (M) and afternoon (A) data of the AN.ON services and comparing that with the results of Tor would unduly favor the AN.ON services, as Tor seems to be much less dependent on daytime.

Anyway, splitting the samples offers another benefit: As described in section 4.1 t-tests operate under the assumption of normally distributed data $10 \mathrm{We}$

${ }_{10}$ Following common practices we use logarithmically transformed values for this purpose. 
found that within each of the two periods the samples are either normally distributed or closely resemble a normal distribution. This is not the case if the samples include data of the whole day, though.

Note that we will only provide results on latencies for the WEB scenarios as they are irrelevant for downloads.

\subsection{Descriptive Statistics for DD, CCC and Tor}

Descriptive statistics can provide some first hints regarding the characteristics of a sample. Our results show that the evaluated systems differ in offered bandwidth and latency. We suspect that the differences are partly due to varying loads (amount of concurrent users) on the anonymisation services. In the rush hours of the afternoon period, DD has very high user numbers (about 1,700 concurrent users on average). In contrast, CCC, which had to be selected manually in order to use it, is used by only 650 users on average. Figure 3 shows the mean values of delay (a) and throughput (b) together with the observed standard deviations for the individual services.

In terms of average delays, CCC offers best performance. The mean values for DD and Tor are considerably worse, but they are too close together for a meaningful graphical comparison. We will provide more concrete results utilising t-tests in section 5.3 and 5.4

On the other hand, Tor might outperform the AN.ON services in terms of bandwidth. Due to the comparably high standard deviations a comparison without thorough analysis is difficult, though. The AN.ON services tend to offer a more constant QoS. From the user perspective, this may be an advantage, as users might not be interested in performance peaks, but rather in adequate performance every time they use the service.

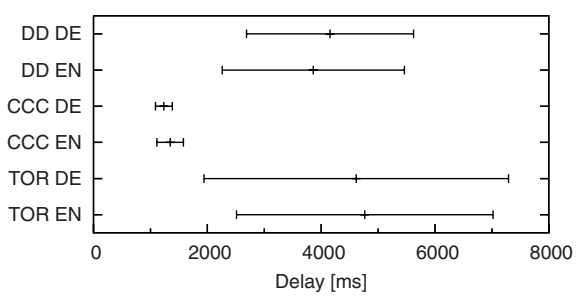

(a) Comparison of delay $(\mathrm{A}, \mathrm{WEB}, \mathrm{EN})$

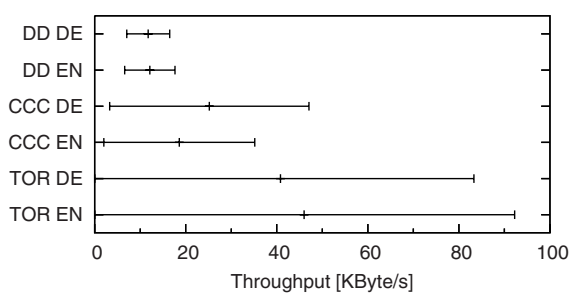

(b) Comparison of throughout $(\mathrm{A}, \mathrm{DL}, \mathrm{EN})$

Fig. 3. Comparison of latency in the afternoon

\subsection{Tor over Daytime}

While performance differences between the morning and afternoon periods are rather obvious for the AN.ON services (cf. figure 6), this is not that clearly visible for Tor. As Tor has a global network of nodes and a distributed user base, this 
is very reasonable. Looking at the descriptive statistics, though, we found that the mean values of delay and throughput differed a lot between the morning and the afternoon period.

The results of the t-test suggest that there is indeed a difference between the two time periods (cf. table 3 and statistical remarks). Local time may therefore have a significant influence on local measurements, and Tor might not only prefer nodes with the highest bandwidth as found in a recent study 4, but also the nearest (low-latency) nodes. This may be due to an implicit attribute of its implementation, although there is no sign of such a strategy in the source code. If so, Tor's practical anonymity would be affected: The difficulty of mounting a collusion attack to capture the connections of specific local user groups would be substantially reduced. Another reason for the observed pattern might be that the initial assumption of a distributed user community is false. This is difficult to prove, though, as the Tor network does not provide information about the location and the number of its users. Accordingly, further research is needed to explain our observations.

Table 3. Tor: Performance differences morning/afternoon

\begin{tabular}{|c|c|c|c|c|c|c|c|c|c|c|}
\hline \multicolumn{3}{|c|}{ Scenario } & \multicolumn{2}{|c|}{ Means (exp) } & \multicolumn{2}{|c|}{ Kol.-Smir. } & \multirow[b]{2}{*}{ Levene } & \multirow{2}{*}{$\frac{\mathrm{T} \text {-Test }}{\mathrm{T}(\mathrm{df})}$} & \multicolumn{2}{|c|}{ Sides } \\
\hline Sim & Lang & Measure & $\mathrm{M}$ & $\mathrm{A}$ & $\mathrm{M}$ & $\mathrm{A}$ & & & 2 & 1 \\
\hline WEB & $\mathrm{DE}$ & Log(Delay) & 3472 & 4097 & - & - & $3.2(177)$ & $-2.3(177)$ & $*$ & $*$ \\
\hline WEB & EN & Log(Delay) & 3790 & 4231 & - & * & $1.0(178)$ & $-1.8(178)$ & - & $*$ \\
\hline WEB & $\mathrm{DE}$ & $\log (T h r)$ & 8.7 & 6.3 & * & - & $6.9(177)^{*}$ & $2.4(170)$ & $* *$ & $* *$ \\
\hline WEB & EN & $\log (\mathrm{Thr})$ & 5.9 & 4.9 & - & - & $0.6(178)$ & $2.1(178)$ & $*$ & $*$ \\
\hline DL & $\mathrm{DE}$ & $\log (T h r)$ & 43.9 & 34.7 & - & - & $0.0(176)$ & $1.9(176)$ & - & $*$ \\
\hline DL & EN & $\log (\mathrm{Thr})$ & 45.7 & 39.1 & - & - & $1.5(176)$ & $1.6(176)$ & - & - \\
\hline
\end{tabular}

Significance codes: ${ }^{* * *} \mathrm{p}<0.001,{ }^{* *} \mathrm{p}<0.01,{ }^{*} \mathrm{p}<0.05$.

Remarks on statistical evaluation. According to the results (cf. rightmost columns of table 3) we have to keep the null hypothesis for half of the scenarios. On the other hand, according to the 1-sided 11 t-test, all scenarios but $\{\mathrm{DL}, \mathrm{EN}\}$ are significant. As the Kolmogorov-Smirnov test is only slightly significant in only two cases, there is a high confidence in the correctness of the test result.

\subsection{Comparison of Tor and DD in the Afternoon}

The DD cascade is the common entry point to the AN.ON system for JAP users. As there is (at the time of measurement) no automatic switching function between different AN.ON cascades, most unexperienced users (who do not know how to switch cascades) use the DD cascade. In terms of latency the statistical

${ }^{11}$ If there is a good reason - not concluded from the collected data - that one of the means should be higher or lower than the other one, the p-value (not shown in the tables) of the t-test may be halved, as only one side of the test is of interest, and the test returns a higher significance. 
results from table 4 show that there is little difference between DD and Tor in the afternoon period. This may indicate that there is a tolerance level for this kind of unexperienced users regarding latency of approximately 4 seconds. A constant latency above this level seems to deter from using the system 12 This supplements the results of [11] who found that there is a linear relation between user numbers and latency by altering the internal delay of the DD service.

Remarks on statistical evaluation. Looking at table 4 we observe that DD seems to have a slight advantage over Tor in regard to latency, but the difference is only significant for the $\{\mathrm{WEB}, \mathrm{EN}\}$ scenario. But then, Tor obviously offers higher channel capacities by far (as shown by the $\{\mathrm{DL}\}$ scenarios) and thus is able to outrun DD in the $\{\mathrm{WEB}\}$ scenarios. The significant difference in bandwidth shows up in the $\{\mathrm{WEB}, \mathrm{EN}\}$ scenario once again: Here, the difference in bandwidth is not as clear as in the $\{\mathrm{WEB}, \mathrm{DE}\}$ scenario however.

Table 4. Comparison of Tor and DD in the afternoon

\begin{tabular}{|c|c|c|c|c|c|c|c|c|c|}
\hline \multicolumn{3}{|c|}{ Scenario } & \multicolumn{2}{|c|}{ Means (exp) } & \multicolumn{2}{|c|}{ Kol.-Smir. } & \multirow[b]{2}{*}{ Levene } & \multicolumn{2}{|c|}{ T-Test } \\
\hline Sim & Lang & Measure & Tor & $\mathrm{DD}$ & Tor & $\mathrm{DD}$ & & $\mathrm{T}(\mathrm{df})$ & Sig \\
\hline WEB & $\mathrm{DE}$ & Log(Delay) & 4032 & 3689 & - & ** & $31.7(178)^{* * *}$ & $-0.3(131)$ & - \\
\hline WEB & $\mathrm{EN}$ & Log(Delay) & 4238 & 3427 & $*$ & $*$ & $19.4(178)^{* * *}$ & $2.1(153)$ & * \\
\hline WEB & $\mathrm{DE}$ & $\log (T h r)$ & 6.30 & 4.30 & - & * & $22.2(178) * * *$ & $3.8(140)$ & $* * *$ \\
\hline WEB & EN & Log(Thr) & 4.92 & 3.75 & - & - & $17.6(178)^{* * *}$ & $2.7(150)$ & $* *$ \\
\hline DL & $\mathrm{DE}$ & $\log (\mathrm{Thr})$ & 34.71 & 10.31 & - & $*$ & $46.1(176)^{* * *}$ & $7.7(119)$ & $* * *$ \\
\hline DL & $\mathrm{EN}$ & Log(Thr) & 39.13 & 10.25 & - & $* * *$ & $53.8(176) * * *$ & $6.7(122)$ & $* * *$ \\
\hline
\end{tabular}

Significance codes: ${ }^{* * *} \mathrm{p}<0.001,{ }^{* *} \mathrm{p}<0.01,{ }^{*} \mathrm{p}<0.05$.

Units: throughput [KBytes/s], delay [msecs].

\subsection{Comparison of Tor and CCC in the Afternoon}

While the DD cascade is the default in AN.ON's client software (JAP), the CCC cascade has to be explicitly selected by the user. Obviously, most users stay with the default (cf. figure 5). Consequently, this situation leads to lower latencies on CCC than on DD. Nevertheless, compared to Tor the bandwith of the CCC cascade is still lagging behind as shown in the $\{\mathrm{DL}\}$ scenarios in table 5. This is true even for the German downloads, where CCC presumably has an implicit advantage. Nevertheless, CCC outperforms Tor in the $\{\mathrm{WEB}\}$ scenarios, which is quite interesting. Apparently, for web surfing extremely low latencies (CCC) are more critical than sheer bandwith (Tor).

\subsection{Correlations of User Numbers and Performance}

In this section we will evaluate the influence of load on performance. AN.ON cascades provide the number of concurrent users at a given time. We will use

$\overline{12}$ Note that using the system and being connected to it are two different perspectives. 
Table 5. Comparison of Tor and CCC in the afternoon

\begin{tabular}{|c|c|c|c|c|c|c|c|c|c|}
\hline \multicolumn{3}{|c|}{ Scenario } & \multicolumn{2}{|c|}{ Means (exp) } & \multicolumn{2}{|c|}{ Kol.-Smir. } & \multirow[b]{2}{*}{ Levene } & \multicolumn{2}{|c|}{ T-Test } \\
\hline Sim & Lang & Measure & Tor & $\mathrm{CCC}$ & Tor & $\mathrm{CCC}$ & & $\mathrm{T}$ (df) & Sig \\
\hline WEB & $\mathrm{DE}$ & Log(Delay) & 4032 & 1091 & - & - & $98.4(178) * * *$ & $17.5(96)$ & $* *$ \\
\hline WEB & EN & Log(Delay) & 4238 & 1191 & * & - & $91.2(178)^{* * *}$ & 18.9(105) & $*$ \\
\hline WEB & $\mathrm{DE}$ & $\log (T h r)$ & 6.30 & 10.07 & - & - & $25.0(178)^{* * *}$ & $-9.1(137)$ & $* * *$ \\
\hline WEB & EN & $\log (\mathrm{Thr})$ & 4.92 & 9.15 & - & - & $23.3(178) * * *$ & $-11.4(143)$ & $* * *$ \\
\hline DL & $\mathrm{DE}$ & $\log (T h r)$ & 34.71 & 21.40 & - & - & $8.4(177)^{* *}$ & $2.4(161)$ & * \\
\hline DL & EN & $\log (T h r)$ & 39.13 & 15.84 & - & ** & $25.0(176)^{* * *}$ & $4.3(142)$ & $* * *$ \\
\hline
\end{tabular}

Significance codes: ${ }^{* * *} \mathrm{p}<0.001,{ }^{* *} \mathrm{p}<0.01,{ }^{*} \mathrm{p}<0.05$.

Units: throughput [KBytes/s], delay [msecs].

this information to investigate the correlation between user number of both AN.ON cascades and the performance parameters. We expect a strong positive correlation between user numbers and latency and a strong negative correlation between user numbers and throughput. Figure 4 shows this graphically in two scatter plots.

The performance parameters have been scaled logarithmically as we expect an exponential influence of the load. The correlation is especially explicit in the selected morning period which contains data points with widely varying user numbers, whereas the afternoon period consists of fairly uniform data that is not suitable for further analysis.

The results of a regression analysis confirm the graphical observations. While both cascades are similar in terms of delay, their characteristics differ a lot in terms of throughput. Apparently, user numbers have a much greater effect on the performance of CCC than on DD. This observation cannot be explained by a gerenally inferior infrastructure (i.e., less capacity) of CCC, which still has plenty of unused resources (cf. figure 3). Instead, we assume that users on DD are considerably less active than those on CCC. A constant and inactive user

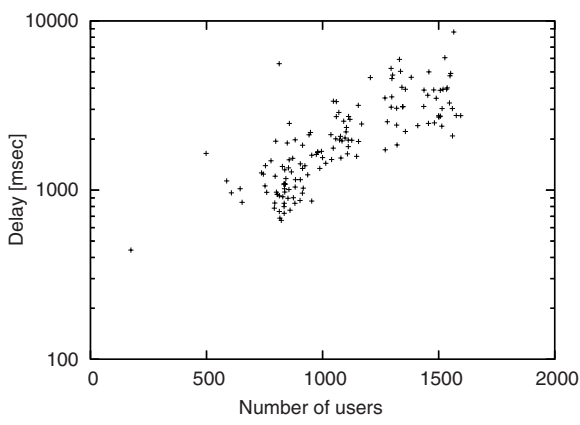

(a) Scatter plot for delay

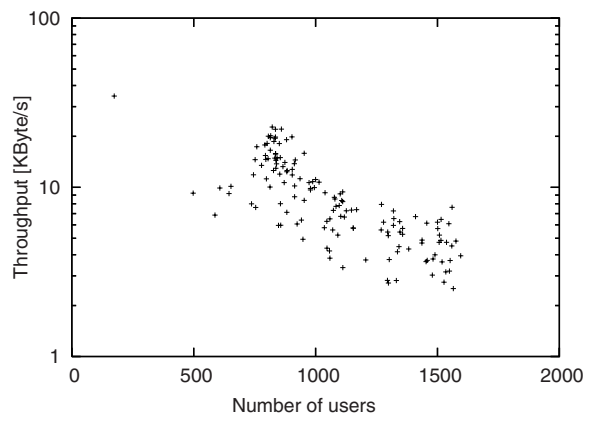

(b) Scatter plot for throughput

Fig. 4. Influence of number of users on performance (M,WEB,DE,DD) 
Table 6. Regression model for performance and user numbers for language DE

\begin{tabular}{|c|c|c|c|c|c|c|}
\hline & \multicolumn{6}{|c|}{ Scenario } \\
\hline & \multicolumn{4}{|c|}{ WEB } & \multicolumn{2}{|c|}{$\mathrm{DL}$} \\
\hline & $\mathrm{DD}$ & $\mathrm{CCC}$ & $\mathrm{DD}$ & $\mathrm{CCC}$ & $\mathrm{DD}$ & CCC \\
\hline Param. $\left(\hat{y}_{i}\right)$ & Log(Delay) & Log(Delay) & $\log (\mathrm{Thr})$ & $\log ($ Thr $)$ & $\log (T h r)$ & $\log (\mathrm{Thr})$ \\
\hline Terms & & & & & & \\
\hline Const. $\left(B_{0}\right)$ & $\begin{array}{c}2.708^{* * * *} \\
(0.04)\end{array}$ & $\begin{array}{c}2.655^{* * *} \\
(0.02)\end{array}$ & $\begin{array}{c}1.49 * * * \\
(0.04)\end{array}$ & $\begin{array}{c}1.66^{* * * *} \\
(0.03)\end{array}$ & $\begin{array}{c}2.11^{* * *} \\
(0.05)\end{array}$ & $\begin{array}{c}2.871^{* * *} \\
(0.05)\end{array}$ \\
\hline Users & $5.075^{* * *}$ & $5.802^{* * *}$ & $-5.215^{* * *}$ & $-10.235^{* * *}$ & $-6.781^{* * *}$ & $-25.170^{* * *}$ \\
\hline Model & & & & & & \\
\hline $\mathrm{N}$ & 258 & 256 & 258 & 256 & 256 & 256 \\
\hline$R^{2}$ & 0.612 & 0.522 & 0.623 & 0.626 & 0.631 & 0.774 \\
\hline $\mathrm{F}$ & $404.2^{* * *}$ & $277.1^{* * *}$ & $422.8^{* * *}$ & $426.0^{* * *}$ & $433.4^{* * *}$ & $870.8^{* * *}$ \\
\hline df & $1 / 256$ & $1 / 254$ & $1 / 256$ & $1 / 255$ & $1 / 255$ & $1 / 255$ \\
\hline
\end{tabular}

Significance codes: $* * * \mathrm{p}<0.001,{ }^{* *} \mathrm{p}<0.01,{ }^{*} \mathrm{p}<0.05$.

Standard errors in brackets (). Users: $B_{1} * 10^{4}$.

base would correspond to the findings in [11] where still some hundred users were counted on DD even when the service had been made unusably slow.

According to these findings raw user numbers are no suitable predictor for load and expected performance on a cascade. We therefore suggest that AN.ON services should only report the number of active users. Otherwise, users might be deceived in terms of the provided anonymity, which is shown in JAP's anonymeter. As adjusted user numbers would correspond to the actual load they could serve as suitable performance measure. Due to their different characteristics finding a uniform regression model for multiple cascades can be a daunting task, though.

Remarks on statistical evaluation. As we assume exponential correlations, all performance parameters are transformed by $\log _{10}$. For the DE scenarios, we could clearly identify normally distributed (transformed) residuals, while this

Table 7. Domains chosen from Alexa's 2] top 20 and Downloads.de/.com top 200

\begin{tabular}{lll}
\hline Simulation Language Domains \\
\hline WEB & DE & $\begin{array}{l}\text { google.de spiegel.de amazon.de t-online.de msn.de mobile.de } \\
\text { leo.org freenet.de arcor.de heise.de } \\
\text { yahoo.com msn.com google.com passport.net amazon.com } \\
\text { myspace.com microsoft.com bbc.co.uk aol.com blogger.com } \\
\text { go.com alibaba.com cnn.com craigslist.org }\end{array}$ \\
& EN & virenschutz.info gratisgames24.de neuesvon.de \\
DL & DE & morpheus.com freewarefiles.com macromedia.com \\
DL & EN &
\end{tabular}

To minimize space requirements, the domains are listed here only, not the downloaded files or the protocol identifier. Files were requested by HTTP only. 
Current users DD

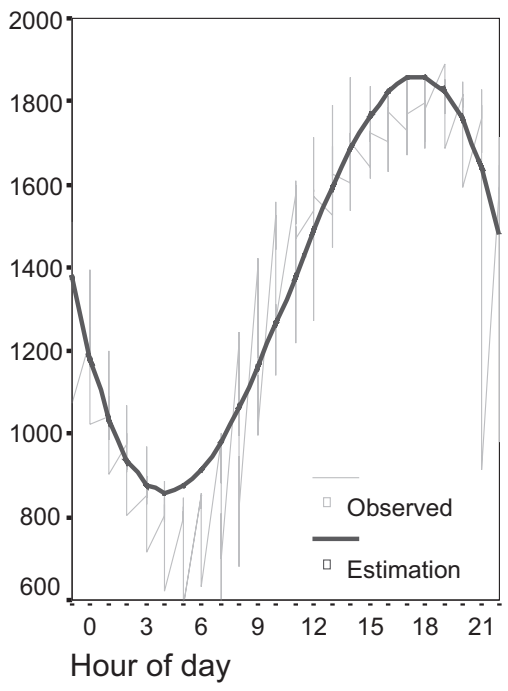

(a) Curve estimation: Avg. users over one day $\{\mathrm{DD}\}$

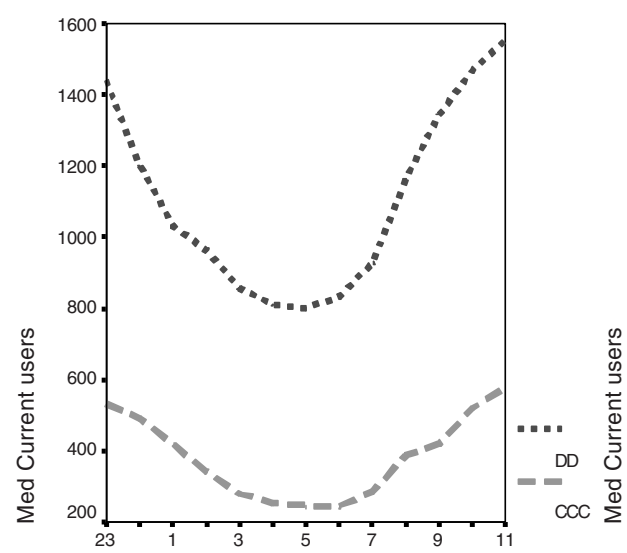

Hour of day

\section{Current users CCC}

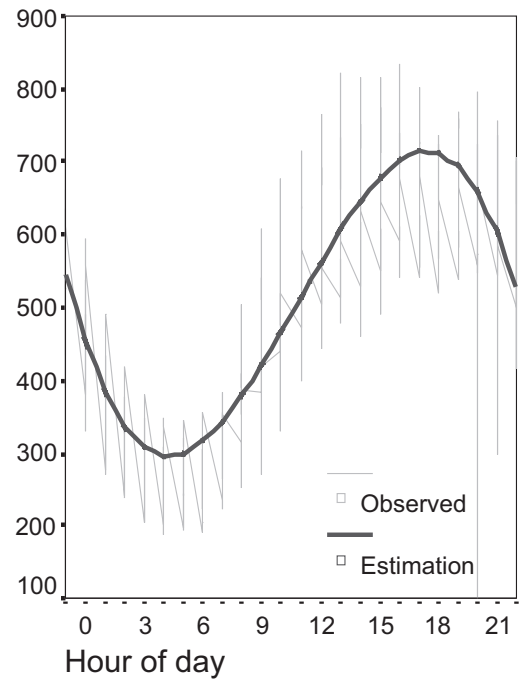

(b) Curve estimation: Avg. users over one day $\{\mathrm{CCC}\}$

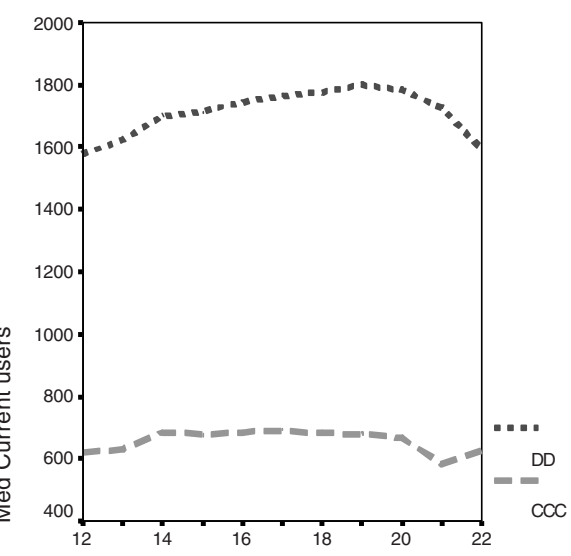

Hour of day

(d) Avg. users from 12 p. m. to 10 p.m.

(c) Avg. users from 11 p.m. to 11 a.m.

Fig. 5. User behaviour in AN.ON cascades

is not the case for the EN scenarios, though. As shown in table 6 the exponential correlation is highly significant and explains most of the spread of the performance parameters $\left(R^{2}>0.5\right)$. 


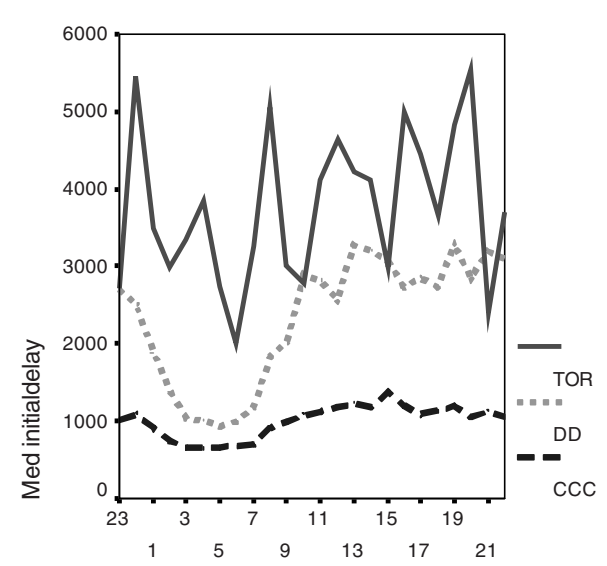

Hour of day

(a) Avg. delay over one day $\{\mathrm{WEB}, \mathrm{EN}\}$

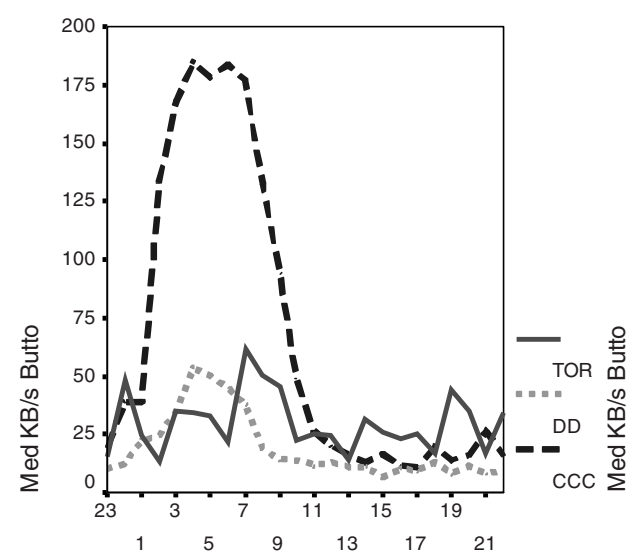

Hour of day

(c) Avg. throughput over one day $\{\mathrm{DL}, \mathrm{DE}\}$

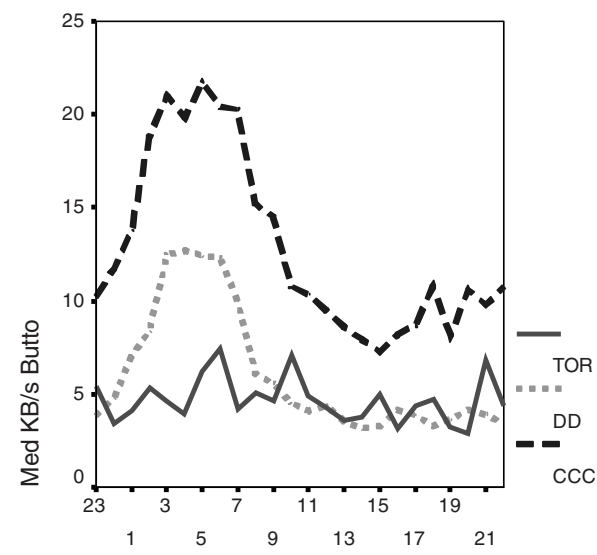

Hour of day

(b) Avg. throughput over one day $\{\mathrm{WEB}, \mathrm{EN}\}$

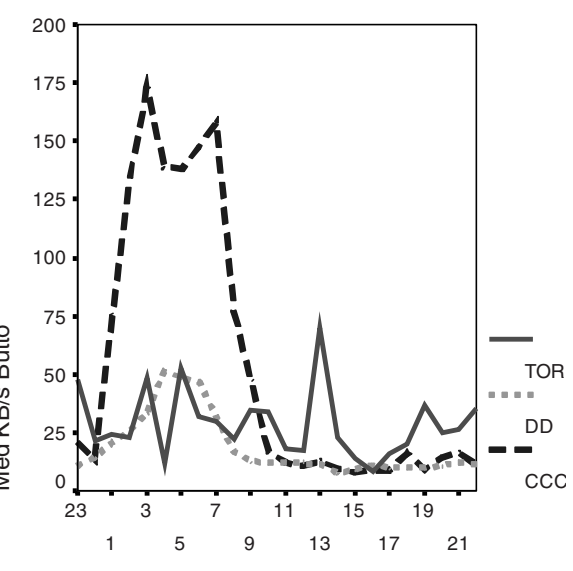

Hour of day

(d) Avg. throughput over one day $\{\mathrm{DL}, \mathrm{EN}\}$

Fig. 6. Graphical comparison of different anonymity services

\section{Future Work}

Maybe our methodology for collecting performance data can be further improved concerning the robustness of the collected data. As measurements took place always in the same interval, this might give rise to inherent biases due to repeated network phenomena being in time with the test cycles. A simple solution might 
Table 8. Data quality measures (cf. section 3.3)

\begin{tabular}{|c|c|c|c|c|c|}
\hline \multirow{2}{*}{$\begin{array}{l}\text { Simulation } \\
\text { Language }\end{array}$} & & \multicolumn{2}{|c|}{ Web browsing } & \multicolumn{2}{|c|}{ Downloads } \\
\hline & & $\mathrm{DE}$ & EN & $\mathrm{DE}$ & $\mathrm{EN}$ \\
\hline Total test cases & & 258 & 258 & 258 & 258 \\
\hline 30 min overlap ratio & & 0.02 & 0.05 & 0.02 & 0.05 \\
\hline 1h breaks & & 0 & 0 & 0 & 0 \\
\hline \multirow{3}{*}{ Critical throughput influence ratio } & TOR & 0.04 & 0.02 & 0.00 & 0.18 \\
\hline & DD & 0.03 & 0.04 & 0.00 & 0.05 \\
\hline & $\mathrm{CCC}$ & 0.13 & 0.28 & 0.01 & 0.22 \\
\hline \multirow{5}{*}{ Missing test cases } & DIRECT & 0 & 1 & 1 & 0 \\
\hline & TOR & 1 & 0 & 2 & 2 \\
\hline & DD & 0 & 1 & 2 & 0 \\
\hline & $\mathrm{CCC}$ & 0 & 0 & 1 & 0 \\
\hline & ALL & 1 & 2 & 6 & 2 \\
\hline Median received KBytes & ALL & 1274.65 & 997.1 & 1529.44 & 1759.69 \\
\hline IQR received KBytes & ALL & 73.64 & 56.50 & 82.00 & 0.00 \\
\hline HTTP Requests w/o failures & \multirow{5}{*}{ DIRECT } & 103130 & 79623 & 771 & 774 \\
\hline Error ratio & & 0.00 & 0.00 & 0.00 & 0.00 \\
\hline Failures & & 0 & 0 & 0 & 0 \\
\hline Median received KBytes ratio & & 0.00 & 0.01 & 0.00 & 0.00 \\
\hline IQR received KBytes ratio & & 0.06 & 0.05 & 0.00 & 0.00 \\
\hline HTTP requests w/o failures & \multirow{5}{*}{ TOR } & 102199 & 79264 & 768 & 768 \\
\hline Error ratio & & 0.00 & 0.00 & 0.02 & 0.02 \\
\hline Failures & & 0 & 0 & 0 & 1 \\
\hline Median received KBytes ratio & & 0.00 & -0.02 & 0.00 & 0.00 \\
\hline IQR received KBytes ratio & & -0.01 & 0.02 & 0.00 & 0.00 \\
\hline HTTP requests $\mathrm{w} / \mathrm{o}$ failures & \multirow{5}{*}{$\mathrm{DD}$} & 102236 & 79200 & 767 & 772 \\
\hline Error ratio & & 0.00 & 0.00 & 0.00 & 0.00 \\
\hline Failures & & 17 & 11 & 15 & 27 \\
\hline Median received KBytes ratio & & 0.00 & 0.00 & 0.00 & 0.00 \\
\hline IQR received KBytes ratio & & 0.06 & 0.17 & 0.00 & 0.00 \\
\hline HTTP requests w/o failures & \multirow{5}{*}{$\mathrm{CCC}$} & 102845 & 79876 & 771 & 774 \\
\hline Error ratio & & 0.00 & 0.00 & 0.00 & 0.09 \\
\hline Failures & & 3 & 0 & 0 & 0 \\
\hline Median received KBytes ratio & & 0.00 & 0.00 & 0.00 & 0.00 \\
\hline IQR received KBytes ratio & & -0.01 & -0.13 & 0.00 & $\infty$ \\
\hline
\end{tabular}

involve randomly changing session time slots or delays (cf. section 3.3), e. g. using a Poisson distribution as proposed in 13 .

Moreover, extending the measured time frame would allow for interesting long term analyses and could help the developer community to understand the impact of newly introduced features. Besides, more AN.ON cascades with high load should be investigated in order to confirm the findings about a user tolerance level, and for building a common regression model for the cascade performance 
depending on user numbers. This will be more promising in the future, as AN.ON now has a client-based load balancing, and may take this study as a reason for only counting active users.

Finally, the time-dependent performance differences of Tor should be further analysed.

\section{Conclusion}

Evaluating the performance of Tor and two AN.ON cascades, we have shown that Tor, a large scale implementation of a free-route mixing protocol, is subject to unpredictable performance, while AN.ON, implementing typically more central mix cascades, is able to offer more consistent performance in general.

The suggestions of the Tor community regarding tuning the connection handling policy of the web browser to mitigate Tor's rather high network latencies 19] are a reasonable approach. Anyway, the overall performance of Tor is already sufficient for fast web surfing and downloads. The reason for the performance differences between morning and afternoon periods remains unclear for now. If Tor's routing strategy was really lured into selecting close-by nodes, this would have considerable implications for the anonymity provided.

In contrast, AN.ON's advantage in latency is restrained by its limited bandwidth and its lack of a load balancing mechanism. Apparently, the DD cascade of AN.ON suffers from high loads (up to 2,000 concurrent users observed). Therefore it cannot deliver satisfying performance during the busy afternoon period where it behaves comparable to Tor regarding latency. The less frequently used CCC cascade is able to offer low-latency web surfing, but at the price of a smaller user base and therefore less anonymity.

An important finding is the supposed user tolerance level for latency: Tor, as a distributed network with many entry points, may automatically adapt to user expectations regarding latency, and therefore pick up as many users as possible with the given network structure. Its performance is not expected to suffer noticeably from single new users connecting to the system. AN.ON, on the other hand, deters a lot of users by offering a single entry point for new users right at the tolerance level, as the performance of this entry point is much more affected by new users than that of Tor.

As this relatively high latency seems to be tolerated by most privacy-aware users, i. e., the ones using Tor or AN.ON, this level may serve as a foundation for a new definition of low-latency in the context of anonymity services. Accordingly, this observation might be useful for designing new and more secure anonymity protocols. Further experiments should verify this level and whether it changes over time.

\section{Acknowledgement}

We thank Rainer Boehme for his priceless help concerning our statistical analysis, the reviewers for their valuable hints and remarks, and Simson Garfinkel for helping us as shepherd to give the article the final cut. 


\section{References}

1. ActiveState ActivePerl: (2006), http://www.activestate.com/Products/ActivePerl/

2. Alexa Top Sites: (2006-02-06), http://www.alexa.com/site/ds/top_sites

3. AN.ON: Protection of Privacy on the Internet (2006), http://www.anon-online.de

4. Bauer, K., et al.: Low-Resource Routing Attacks Against Anonymous Systems. Technical Report (2007), http://www.cs.colorado.edu/department/publications/reports/docs/ CU-CS-1025-07.pdf

5. Boehme, R., et al.: On the PET Workshop Panel Mix Cascades vs. Peer-to-Peer: Is One Concept Superior? In: Martin, D., Serjantov, A. (eds.) PET 2004. LNCS, vol. 3424, pp. 243-255. Springer, Heidelberg (2005)

6. Chaum, D.: Untraceable electronic mail, return addresses, and digital pseudonyms. Communications of the ACM 4(2) (1981)

7. Draper, N.R., et al.: Applied Regression Analysis, p. 17. Wiley, New York (1966)

8. Federrath, H.: Privacy Enhanced Technologies: Methods - Markets - Misuse. In: Katsikas, S.K., Lopez, J., Pernul, G. (eds.) TrustBus 2005. LNCS, vol. 3592, pp. 1-9. Springer, Heidelberg (2005)

9. I2P: (2006), http://www.i2p.net

10. JMeter: (2006), http://jakarta.apache.org/jmeter/

11. Köpsell, S.: Low Latency Anonymous Communication - How long are users willing to wait? In: Müller, G. (ed.) ETRICS 2006. LNCS, vol. 3995, pp. 221-237. Springer, Heidelberg (2006)

12. LWP: ParallelUA 2.57 (2006), http://search.cpan.org/ marclang/ParallelUserAgent-2.57/

13. Paxson, V.: End-to-end routing behavior in the internet. In: Proceedings of the ACM SIGCOMM Conference on Applications, Technologies, Architectures, and Protocols for Computer Communications, pp. 25-38 (1996)

14. RFC2616 Hypertext Transfer Protocol - HTTP/1.1. Section 14.9 (2006)

15. Servertest: (2006), http://softwaregarden.com/products/servertest/index.html

16. Sheskin, D.J.: Handbook of parametric and nonparametric statistical procedures, 2nd edn., p. 247. Chapman \& Hall/CRC, Boca Raton (2000)

17. Tor: An anonymous Internet communication system (2006), http://tor.eff.org

18. Tor FAQ: Why does Google show up in foreign languages? (2006), http://wiki.noreply.org/noreply/TheOnionRouter/TorFAQ\#GoogleLanguage

19. Tor Wiki: (2006), http://wiki.noreply.org/noreply/TheOnionRouter/FireFoxTorPerf

\section{Appendix}

The criteria for our choices of URLs were

- server performance much better than performance offered by anonymisation service, so that the results are not biased by slow servers 13

${ }^{13}$ As we can never be sure that all servers have an adequate speed during the measurement, we aggregate the download performance of a set of URLs to a test case in order to mitigate possible influences. 
- comparable number of URLs and downloaded bytes within the same scenario

- low number of HTTP errors produced by the requested web servers

- average total download time for web sites plus downloads of one language is much smaller than 30 minutes

- for web site URLs: plausibility of ranking in the Alexa top list

Geolocation detection. As stated in section 3.3 the separation of the EN/DE scenarios might be jeopardised through geolocation of the client based on its IP address. Geolocation is performed by the webserver in order to (1) provide a localised version of a web site, or to (2) enhance the user-view performance by redirecting the request to a "nearer" webserver.

Localised versions of web sites do not influence our tests unduly, because latency and bandwidth are not affected. However, if requests are re-routed to another server, this will change. We applied the following checks to check whether any form of request re-routing took place:

- We utilised the Unix dig utility and examined the DNS records for the individual hosts. We found multiple IPs and short TTLs, which indicates that several sites employed round robin IP rotation. Typically, web sites under high load use this approach for load balancing, but not for geolocation.

- We requested the individual URLs from our $\{\mathrm{EN}\}$ scenarios with the Unix wget utility and looked for HTTP redirects, which the webserver might send during geolocation: No URL used in the scenarios employed HTTP redirects for their homepage. 